Supporting Information

\title{
Sphingolipidomics investigation of the temporal dynamics after ischemic brain
} injury

Hsi-Chun Chao ${ }^{\dagger, t}$, Tsung-Heng Lee ${ }^{\dagger, t}$, Chien-Sung Chiang ${ }^{\S}$, Sin-Yu Yang ${ }^{\S}$, Ching-Hua Kuo ${ }^{\dagger, t, \perp, *}$, and Sung-Chun Tang ${ }^{\S, *}$

$\dagger$ School of Pharmacy, College of Medicine, National Taiwan University, Taipei, Taiwan

$\mp$ The Metabolomics Core Laboratory, Center of Genomic Medicine, National Taiwan University, Taipei, Taiwan

$\S$ Stroke Center and Department of Neurology, National Taiwan University Hospital, Taipei, Taiwan

$\perp$ Department of Pharmacy, National Taiwan University Hospital, Taipei, Taiwan

*Corresponding Author

Ching-Hua Kuo

Address: No.33, Linsen S. Rd., Taipei 100, Taiwan

Tel.: +886.2 .33668766$

E-mail: kuoch@,ntu.edu.tw

Sung-Chun Tang

Address: No.7, Chung-Shan S.Rd., Taipei 100, Taiwan.

Tel.: +886.2.23563279

E-mail:sctang@,ntuh.gov.tw 


\section{This SI contains the following:}

Supplementary protocol page S-3

Supplementary Figure 1. Bar Charts which compares the fold changes of individual ceramide on primary neuronal cultured cells in response to OGD treatment at 0,3 and 24 hr. page S-4

Supplementary Figure 2. The neurological scores evaluated at different time points after MCAO treatment and with or without atorvastatin treatment. **: p-value $<0.01 ; *$ : p-value $<0.05$. (Score definitions: 0 , no deficit; 1 , failure to extend right paw; 2 , circling to the right; 3 , falling to the right; and 4 , inability to walk spontaneously.) .page S-4

Supplementary Figure 3. The bar charts for phosphocholine on primary neuronal cultured cells in response to OGD treatment at 3 and $24 \mathrm{hr} .^{*}: \mathrm{p}$-value $<0.05$. page S-5

Supplementary Table 1. Molecule weight and retention time library for the sphingolipidomic analysis ..page S-6

Supplementary Table 2. Extraction recoveries for different sphingolipids page S-9 


\section{Supplementary protocol}

\section{QC sample preparation and analysis}

The QC samples were prepared after sample extraction. $10 \mu \mathrm{L}$ of aliquot from reconstituted samples was collected and mixed to form QC sample. QC sample was injected every 6 samples and used for evaluated the stability of the instrument. The 47 different sphingolipids were all detected in the QC samples and the RSD of integrated area from each peak was all within $10 \%$.

\section{Reconstitution solvent preparation (with internal standard)}

The internal standard stock solution was prepared by dissolving $1.0 \mathrm{mg}$ Cer (d18:1/17:0) in $1.000 \mathrm{~mL} 100 \% \mathrm{MS}$-graded $\mathrm{MeOH}$. The stock solution was further diluted to $100 \mathrm{ng} / \mathrm{mL}$ to prepare the reconstitution solvent with $100 \% \mathrm{MeOH}$.

\section{Neurological score evaluation}

The functional consequences of $\mathrm{I} / \mathrm{R}$ injury were evaluated using a 5-point neurological deficit score described in our previous study ( 0 , no deficit; 1 , failure to extend right paw; 2 , circling to the right; 3 , falling to the right; and 4, inability to walk spontaneously) ${ }^{1}$. 


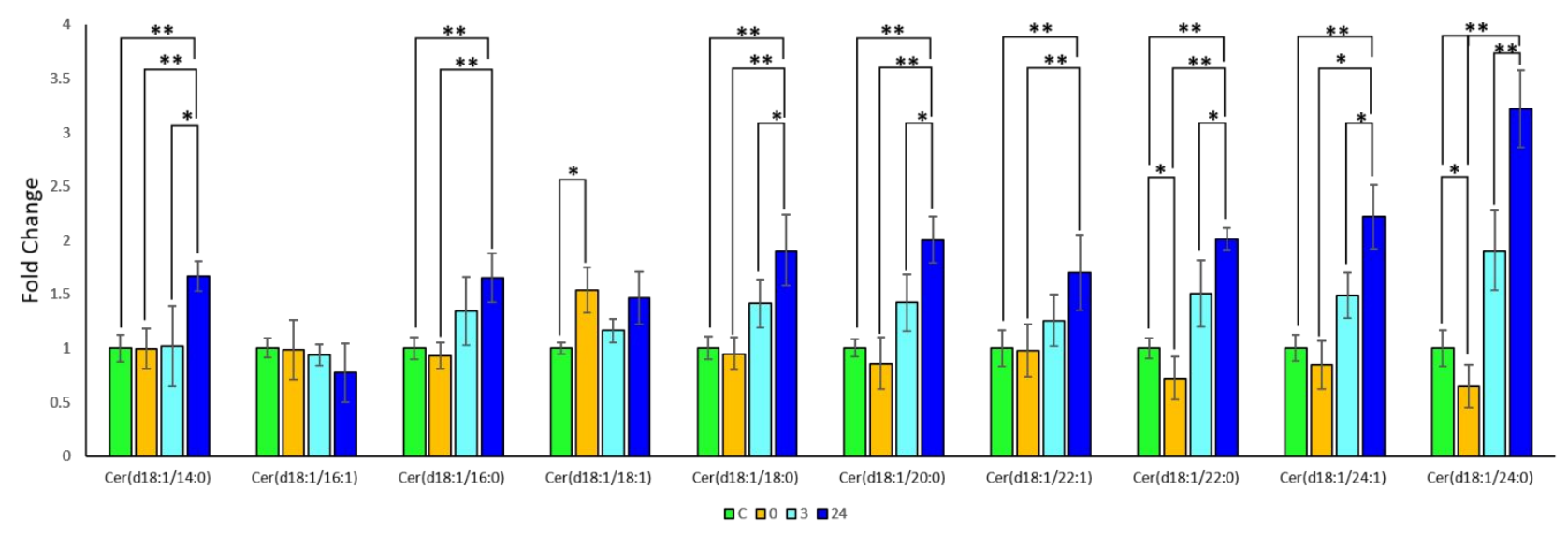

Supplementary Figure 1. Bar Charts which compares the fold changes of individual ceramide on primary neuronal cultured cells in response to OGD treatment at 0,3 and $24 \mathrm{hr}$.

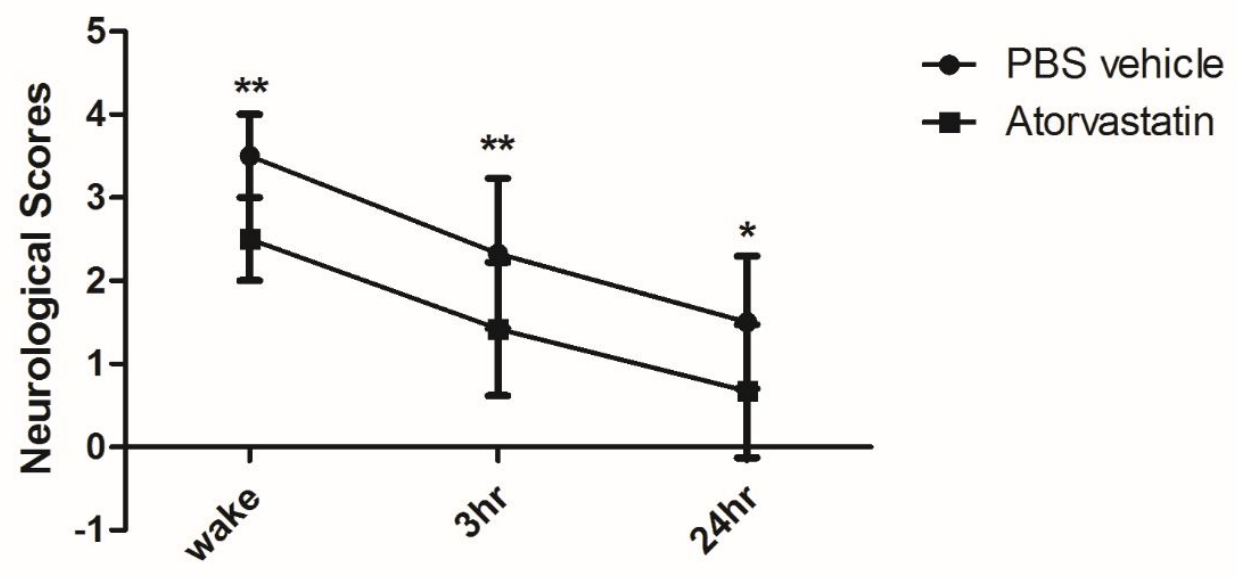

Supplementary Figure 2. The neurological scores evaluated at different time points after MCAO treatment and with or without atorvastatin treatment. **: p-value $<0.01 ; *$ : p-value $<0.05$. (Score definitions: 0 , no deficit; 1 , failure to extend right paw; 2 , circling to the right; 3 , falling to the right; and 4, inability to walk spontaneously.) 


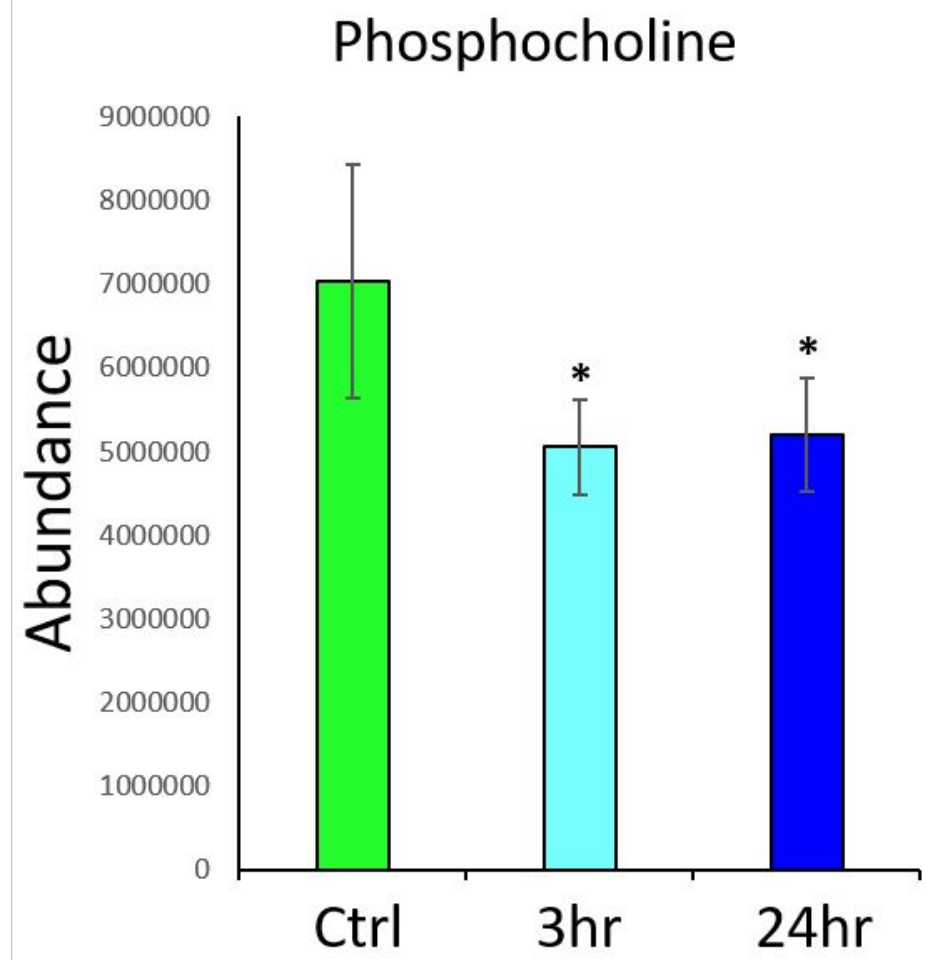

Supplementary Figure 3. The bar charts for phosphocholine on primary neuronal cultured cells in response to OGD treatment at 3 and $24 \mathrm{hr} .^{*}$ : p-value $<0.05$. 
Supplementary Table 1. Molecule weight and retention time library for the sphingolipidomic analysis ${ }^{2}$.

\begin{tabular}{|c|c|c|c|c|}
\hline \multicolumn{5}{|l|}{ Sphingolipid base } \\
\hline Name & formula & {$[\mathbf{M}+\mathbf{H}]^{+}$} & Product ion & RT (min) \\
\hline \multirow[t]{2}{*}{ Sphingosine } & $\mathrm{C} 18 \mathrm{H} 37 \mathrm{NO} 2$ & 300.3 & 282.3 & 1.837 \\
\hline & & 300.3 & 55 & \\
\hline \multirow[t]{2}{*}{ Sphinganine } & $\mathrm{C} 18 \mathrm{H} 39 \mathrm{NO} 3$ & 302.3 & 284.3 & 2.074 \\
\hline & & 302.3 & 60.1 & \\
\hline \multirow[t]{2}{*}{ Sphingosine-1-phosphate } & C18H38NO5P & 380.3 & 264.2 & 2.263 \\
\hline & & 380.3 & 82 & \\
\hline \multirow[t]{2}{*}{ Sphinganine-1-phospahte } & $\mathrm{C} 18 \mathrm{H} 40 \mathrm{NO} 5 \mathrm{P}$ & 382.3 & 284.3 & 2.568 \\
\hline & & 382.3 & 60.1 & \\
\hline \multicolumn{5}{|l|}{ Ceramides } \\
\hline Name & formula & {$[\mathbf{M}+\mathbf{H}]^{+}$} & Product ion & RT (min) \\
\hline Cer(d18:1/14:0) & C32H63NO3 & 510.5 & 264.3 & 7.125 \\
\hline Cer(d18:1/15:0) & C33H65NO3 & 524.5 & 264.3 & 7.502 \\
\hline Cer(d18:1/16:1) & C34H65NO3 & 536.5 & 264.3 & 7.614 \\
\hline $\operatorname{Cer}(\mathrm{d} 18: 1 / 16: 0)$ & $\mathrm{C} 34 \mathrm{H} 67 \mathrm{NO} 3$ & 538.5 & 264.3 & 8.104 \\
\hline $\operatorname{Cer}(\mathrm{d} 18: 1 / 17: 1)$ & $\mathrm{C} 35 \mathrm{H} 67 \mathrm{NO} 3$ & 550.5 & 264.3 & 9.252 \\
\hline $\operatorname{Cer}(\mathrm{d} 18: 1 / 17: 0)$ & C35H69NO3 & 552.5 & 264.3 & 8.634 \\
\hline $\operatorname{Cer}(\mathrm{d} 18: 1 / 18: 0)$ & $\mathrm{C} 36 \mathrm{H} 71 \mathrm{NO} 3$ & 566.5 & 264.3 & 9.26 \\
\hline Cer(d18:1/19:0) & C37H73NO3 & 580.6 & 264.3 & 9.934 \\
\hline Cer(d18:1/20:1) & C38H73NO3 & 592.5 & 264.3 & 8.995 \\
\hline Cer(d18:1/20:0) & C38H75NO3 & 594.6 & 264.3 & 10.496 \\
\hline $\operatorname{Cer}(\mathrm{d} 18: 1 / 21: 0)$ & C39H77NO3 & 608.6 & 264.3 & 11.203 \\
\hline Cer(d18:1/22:1) & $\mathrm{C} 40 \mathrm{H} 77 \mathrm{NO} 3$ & 620.5 & 264.3 & 10.127 \\
\hline $\operatorname{Cer}(\mathrm{d} 18: 1 / 22: 0)$ & $\mathrm{C} 40 \mathrm{H} 79 \mathrm{NO} 3$ & 622.6 & 264.3 & 12.014 \\
\hline $\operatorname{Cer}(\mathrm{d} 18: 1 / 23: 1)$ & C41H79NO3 & 634.6 & 264.3 & 11.404 \\
\hline Cer(d18:1/23:0) & $\mathrm{C} 41 \mathrm{H} 81 \mathrm{NO} 3$ & 636.6 & 264.3 & 12.945 \\
\hline Cer(d18:1/24:1) & $\mathrm{C} 42 \mathrm{H} 81 \mathrm{NO} 3$ & 648.6 & 264.3 & 12.209 \\
\hline Cer(d18:1/24:0) & C42H83NO3 & 650.6 & 264.3 & 13.948 \\
\hline Cer(d18:1/25:1) & $\mathrm{C} 43 \mathrm{H} 83 \mathrm{NO} 3$ & 662.7 & 264.3 & 13.106 \\
\hline
\end{tabular}




\begin{tabular}{|c|c|c|c|c|}
\hline Cer(d18:1/25:0) & $\mathrm{C} 43 \mathrm{H} 85 \mathrm{NO} 3$ & 664.7 & 264.3 & 14.751 \\
\hline $\operatorname{Cer}(\mathrm{d} 18: 1 / 26: 1)$ & C44H85NO3 & 676.7 & 264.3 & 14.085 \\
\hline Cer(d18:1/26:0) & C44H87NO3 & 678.7 & 264.3 & 15.434 \\
\hline Cer(d18:2/16:0) & C34H65NO3 & 536.5 & 262.3 & 7.39 \\
\hline $\operatorname{Cer}(\mathrm{d} 18: 2 / 18: 1)$ & C36H67NO3 & 562.5 & 262.3 & 7.687 \\
\hline Cer(d18:2/18:0) & C36H69NO3 & 564.6 & 262.3 & 8.401 \\
\hline Cer(d18:2/19:0) & $\mathrm{C} 37 \mathrm{H} 71 \mathrm{NO} 3$ & 578.5 & 262.3 & 8.979 \\
\hline $\operatorname{Cer}(\mathrm{d} 18: 2 / 20: 1)$ & C38H71NO3 & 590.5 & 262.3 & 8.152 \\
\hline Cer(d18:2/20:0) & $\mathrm{C} 38 \mathrm{H} 73 \mathrm{NO} 3$ & 592.6 & 262.3 & 9.621 \\
\hline Cer(d18:2/21:0) & C39H75NO3 & 606.6 & 262.3 & 10.24 \\
\hline $\operatorname{Cer}(\mathrm{d} 18: 2 / 22: 0)$ & $\mathrm{C} 40 \mathrm{H} 77 \mathrm{NO} 3$ & 620.6 & 262.3 & 10.914 \\
\hline Cer(d18:2/23:0) & C41H79NO3 & 634.6 & 262.3 & 11.644 \\
\hline Cer(d18:2/24:1) & $\mathrm{C} 42 \mathrm{H} 79 \mathrm{NO} 3$ & 646.6 & 262.3 & 11.066 \\
\hline Cer(d18:2/24:0) & $\mathrm{C} 42 \mathrm{H} 81 \mathrm{NO} 3$ & 648.6 & 262.3 & 12.536 \\
\hline $\operatorname{Cer}(\mathrm{d} 18: 2 / 25: 0)$ & C43H83NO3 & 662.7 & 262.3 & 13.579 \\
\hline $\operatorname{Cer}(\mathrm{d} 18: 2 / 26: 1)$ & $\mathrm{C} 44 \mathrm{H} 83 \mathrm{NO} 3$ & 674.7 & 262.3 & 12.608 \\
\hline Cer(d18:2/26:0) & $\mathrm{C} 44 \mathrm{H} 85 \mathrm{NO} 3$ & 676.7 & 262.3 & 14.438 \\
\hline \multicolumn{5}{|l|}{ Dihydroceramides } \\
\hline Name & formula & {$[\mathbf{M}+\mathbf{H}]^{+}$} & Product ion & RT (min) \\
\hline DihydroCer(d18:0/16:0) & C34H69NO3 & 540.5 & 266.3 & 7.948 \\
\hline DihydroCer(d18:0/18:0) & $\mathrm{C} 36 \mathrm{H} 73 \mathrm{NO} 3$ & 568.6 & 266.3 & 9.284 \\
\hline DihydroCer (d18:0/20:0) & $\mathrm{C} 38 \mathrm{H} 77 \mathrm{NO} 3$ & 596.6 & 266.3 & 11.026 \\
\hline DihydroCer (d18:0/22:0) & $\mathrm{C} 40 \mathrm{H} 81 \mathrm{NO} 3$ & 624.6 & 266.3 & 12.022 \\
\hline DihydroCer (d18:0/24:1) & $\mathrm{C} 42 \mathrm{H} 83 \mathrm{NO} 3$ & 650.6 & 266.3 & 12.23 \\
\hline DihydroCer (d18:0/24:0) & $\mathrm{C} 42 \mathrm{H} 85 \mathrm{NO} 3$ & 652.6 & 266.3 & 14.013 \\
\hline \multicolumn{5}{|l|}{ Cerebrosides } \\
\hline Name & formula & {$[\mathbf{M}+\mathbf{H}]^{+}$} & Product ion & RT (min) \\
\hline $\mathrm{CB}(\mathrm{d} 18: 1 / 16: 0)$ & $\mathrm{C} 40 \mathrm{H} 77 \mathrm{NO} 8$ & 700.6 & 264.3 & 7.403 \\
\hline $\mathrm{CB}(\mathrm{d} 18: 1 / 17: 0)$ & $\mathrm{C} 41 \mathrm{H} 79 \mathrm{NO} 8$ & 714.6 & 264.3 & 7.903 \\
\hline $\mathrm{CB}(\mathrm{d} 18: 1 / 18: 1)$ & $\mathrm{C} 42 \mathrm{H} 79 \mathrm{NO} 8$ & 726.6 & 264.3 & 8.141 \\
\hline $\mathrm{CB}(\mathrm{d} 18: 1 / 18: 0)$ & $\mathrm{C} 42 \mathrm{H} 81 \mathrm{NO} 8$ & 728.6 & 264.3 & 8.428 \\
\hline $\mathrm{CB}(\mathrm{d} 18: 1 / 19: 1)$ & $\mathrm{C} 43 \mathrm{H} 81 \mathrm{NO} 8$ & 740.6 & 264.3 & 8.675 \\
\hline $\mathrm{CB}(\mathrm{d} 18: 1 / 19: 0)$ & $\mathrm{C} 43 \mathrm{H} 83 \mathrm{NO} 8$ & 742.6 & 264.3 & 9.06 \\
\hline
\end{tabular}




\begin{tabular}{|c|c|c|c|c|}
\hline $\mathrm{CB}(\mathrm{d} 18: 1 / 20: 1)$ & $\mathrm{C} 44 \mathrm{H} 83 \mathrm{NO} 8$ & 754.6 & 264.3 & 9.257 \\
\hline CB(d18:1/20:0) & C44H85NO8 & 756.6 & 264.3 & 9.577 \\
\hline $\mathrm{CB}(\mathrm{d} 18: 1 / 21: 1)$ & $\mathrm{C} 45 \mathrm{H} 85 \mathrm{NO} 8$ & 768.6 & 264.3 & 9.807 \\
\hline $\mathrm{CB}(\mathrm{d} 18: 1 / 21: 0)$ & $\mathrm{C} 45 \mathrm{H} 87 \mathrm{NO} 8$ & 770.6 & 264.3 & 10.168 \\
\hline $\mathrm{CB}(\mathrm{d} 18: 1 / 22: 1)$ & C46H87NO8 & 782.7 & 264.3 & 10.439 \\
\hline CB(d18:1/22:0) & C46H89NO8 & 784.7 & 264.3 & 10.816 \\
\hline CB(d18:1/23:1) & C47H89NO8 & 796.7 & 264.3 & 11.12 \\
\hline CB(d18:1/23:0) & C47H91NO8 & 798.7 & 264.3 & 11.552 \\
\hline $\mathrm{CB}(\mathrm{d} 18: 1 / 24: 1)$ & C48H91NO8 & 810.7 & 264.3 & 11.875 \\
\hline $\mathrm{CB}(\mathrm{d} 18: 1 / 24: 0)$ & C48H93NO8 & 812.7 & 264.3 & 12.351 \\
\hline $\mathrm{CB}(\mathrm{d} 18: 1 / 25: 1)$ & C49H93NO8 & 824.7 & 264.3 & 12.737 \\
\hline CB(d18:1/25:0) & C49H95NO8 & 826.7 & 264.3 & 13.303 \\
\hline $\mathrm{CB}(\mathrm{d} 18: 1 / 26: 1)$ & C50H95NO8 & 838.7 & 264.3 & 13.795 \\
\hline CB(d18:1/26:0) & C50H97NO8 & 840.7 & 264.3 & 14.255 \\
\hline \multicolumn{5}{|c|}{ Ceramide-1-phosphate } \\
\hline Name & formula & {$[\mathbf{M}+\mathbf{H}]^{+}$} & Product ion & RT (min) \\
\hline $\operatorname{CerP}(\mathrm{d} 18: 1 / 12: 0)$ & C30H60NO6P & 562.4 & 264.3 & 5.211 \\
\hline $\operatorname{CerP}(\mathrm{d} 18: 1 / 16: 0)$ & C34H68NO6P & 618.5 & 264.3 & 6.532 \\
\hline $\operatorname{CerP}(\mathrm{d} 18: 1 / 18: 1)$ & C36H70NO6P & 644.5 & 264.3 & 6.729 \\
\hline CerP(d18:1/24:0) & C42H84NO6P & 730.6 & 264.3 & 10.525 \\
\hline \multicolumn{5}{|l|}{ Sphingomyelin } \\
\hline Name & formula & {$[\mathbf{M}+\mathbf{H}]^{+}$} & Product ion & RT (min) \\
\hline $\operatorname{SM}(\mathrm{d} 18: 1 / 14: 0)$ & C37H75N2O6P & 675.5 & 184.3 & 6.192 \\
\hline $\mathrm{SM}(\mathrm{d} 18: 1 / 14: 1)$ & $\mathrm{C} 37 \mathrm{H} 73 \mathrm{~N} 2 \mathrm{O} 6 \mathrm{P}$ & 673.5 & 184.3 & 5.733 \\
\hline $\operatorname{SM}(\mathrm{d} 18: 1 / 15: 0)$ & C38H77N2O6P & 689.6 & 184.3 & 6.585 \\
\hline $\operatorname{SM}(\mathrm{d} 18: 1 / 16: 0)$ & C39H79N2O6P & 703.6 & 184.3 & 7.011 \\
\hline $\operatorname{SM}(\mathrm{d} 18: 1 / 16: 1)$ & C39H77N2O6P & 701.6 & 184.3 & 6.404 \\
\hline $\operatorname{SM}(\mathrm{d} 18: 1 / 17: 0)$ & C40H81N2O6P & 717.6 & 184.3 & 7.477 \\
\hline $\mathrm{SM}(\mathrm{d} 18: 1 / 17: 1)$ & $\mathrm{C} 40 \mathrm{H} 79 \mathrm{~N} 2 \mathrm{O} 6 \mathrm{P}$ & 715.6 & 184.3 & 6.798 \\
\hline SM(d18:1/18:0) & C41H83N2O6P & 731.6 & 184.3 & 7.952 \\
\hline $\operatorname{SM}(d 18: 1 / 18: 1)$ & C41H81N2O6P & 729.6 & 184.3 & 7.314 \\
\hline $\mathrm{SM}(\mathrm{d} 18: 1 / 19: 0)$ & $\mathrm{C} 42 \mathrm{H} 85 \mathrm{~N} 2 \mathrm{O} 6 \mathrm{P}$ & 745.6 & 184.3 & 8.468 \\
\hline $\operatorname{SM}(\mathrm{d} 18: 1 / 20: 0)$ & C43H87N2O6P & 759.6 & 184.3 & 9.052 \\
\hline
\end{tabular}




\begin{tabular}{|l|l|l|l|c|}
\hline $\mathrm{SM}(\mathrm{d} 18: 1 / 20: 1)$ & C43H85N2O6P & 757.6 & 184.3 & 8.198 \\
\hline $\mathrm{SM}(\mathrm{d} 18: 1 / 21: 0)$ & C44H89N2O6P & 773.7 & 184.3 & 9.648 \\
\hline $\mathrm{SM}(\mathrm{d} 18: 1 / 22: 0)$ & C45H91N2O6P & 787.7 & 184.3 & 10.237 \\
\hline $\mathrm{SM}(\mathrm{d} 18: 1 / 22: 1)$ & $\mathrm{C} 45 \mathrm{H} 89 \mathrm{~N} 2 \mathrm{O} 6 \mathrm{P}$ & 785.7 & 184.3 & 9.205 \\
\hline $\mathrm{SM}(\mathrm{d} 18: 1 / 23: 0)$ & $\mathrm{C} 46 \mathrm{H} 93 \mathrm{~N} 2 \mathrm{O} 6 \mathrm{P}$ & 801.7 & 184.3 & 10.86 \\
\hline $\mathrm{SM}(\mathrm{d} 18: 1 / 24: 0)$ & $\mathrm{C} 47 \mathrm{H} 95 \mathrm{~N} 2 \mathrm{O} 6 \mathrm{P}$ & 815.7 & 184.3 & 11.597 \\
\hline $\mathrm{SM}(\mathrm{d} 18: 1 / 24: 1)$ & $\mathrm{C} 47 \mathrm{H} 93 \mathrm{~N} 2 \mathrm{O} 6 \mathrm{P}$ & 813.7 & 184.3 & 10.344 \\
\hline $\mathrm{SM}(\mathrm{d} 18: 1 / 25: 0)$ & $\mathrm{C} 48 \mathrm{H} 95 \mathrm{~N} 2 \mathrm{O} 6 \mathrm{P}$ & 829.7 & 184.3 & 12.432 \\
\hline
\end{tabular}

Supplementary Table 2. Extraction recoveries of different sphingolipids. $(n=3)$

\begin{tabular}{ccc}
\hline Name & Concentration $(\mathbf{n g} / \mathbf{m L})$ & Recovery $\mathbf{( \% )}$ \\
\hline Sphingosine (d18:1) & 0.100 & $84.54 \pm 10.27$ \\
& 10.00 & $41.58 \pm 4.45$ \\
Sphingosine (d18:1) & 1,000 & $57.62 \pm 1.51$ \\
\hline SM (d18:1/18:0) & 0.100 & $47.30 \pm 5.60$ \\
& 10.00 & $43.10 \pm 3.48$ \\
& 1,000 & $49.61 \pm 1.36$ \\
\hline Cer (d18:1/18:0) & 0.100 & $76.86 \pm 13.49$ \\
& 10.00 & $85.62 \pm 8.61$ \\
& 1,000 & $92.94 \pm 0.91$ \\
\hline CB (d18:1/18:0) & 0.100 & $109.75 \pm 9.39$ \\
& 10.00 & $74.76 \pm 7.49$ \\
& 1,000 & $95.47 \pm 2.62$ \\
\hline & 0.100 & $78.49 \pm 9.31$ \\
\hline
\end{tabular}

$*$ Mean \pm SD. 
Ref:

1. Tang, S.-C.; Wang, Y.-C.; Li, Y.-I.; Lin, H.-C.; Manzanero, S.; Hsieh, Y.-H.; Phipps, S.; Hu, C.-J.; Chiou, H.-Y.; Huang, Y.-S.; Yang, W.-S.; Mattson, M. P.; Arumugam, T. V.; Jeng, J.-S., Functional Role of Soluble Receptor for Advanced Glycation End Products in Stroke.

Arteriosclerosis, Thrombosis, and Vascular Biology 2013, 33 (3), 585-594.

2. Chao, H.-C.; Chen, G.-Y.; Hsu, L.-C.; Liao, H.-W.; Yang, S.-Y.; Wang, S.-Y.; Li, Y.-L.; Tang, S.-C.; Tseng, Y. J.; Kuo, C.-H., Using precursor ion scan of 184 with liquid chromatographyelectrospray ionization-tandem mass spectrometry for concentration normalization in cellular lipidomic studies. Anal Chim Acta 2017, 971, 68-77. 